\title{
Rapid procedure to calibrate EC-10 and EC-20 capacitance sensors in coir
}

\author{
RJ van der Westhuizen' and LD van Rensburg' ${ }^{2 *}$ \\ 'Department of Agronomy, University of Stellenbosch, Private Bag X1, Matieland 7602, South Africa \\ ${ }^{2}$ Department of Soil, Crop and Climate Sciences, University of the Free State, PO Box 339, Bloemfontein 9300, South Africa
}

\section{ABSTRACT}

\begin{abstract}
A rapid calibration procedure for EC-10 and EC-20 sensors is introduced to promote the commercial use of these sensors for hydroponic irrigation management in coir. The method is comprised of taking one sensor reading, by a sensor installed under hydroponic crop production conditions, and one gravimetric sample, both at field water capacity, to determine a correction factor for an accurate general laboratory calibration equation developed for coir. The rapid calibration procedure was evaluated by 4 separate sensors of EC-10 and EC-20. To verify the relative reliability of the rapid procedure, statistical analysis was performed separately for all data points and for data points between the drained upper limit and permanent wilting point of coir. From the statistical parameters used, it was observed that all of the predictions in the plant available water content range were good, with RMSE values $<0.030 \mathrm{~m}^{3} \cdot \mathrm{m}^{-3}$ for the EC-10 and $<0.021 \mathrm{~m}^{3} \cdot \mathrm{m}^{-3}$ for the EC-20 sensors. The D-index also pointed to a high accuracy of prediction in the plant available water content range, with values over 0.981 and 0.990 for the EC-10 and EC-20 sensors, respectively. Since a degree of variation remained between sensors, it was concluded that sensors should be calibrated individually. The rapid procedure proves a simple but scientifically sound method to calibrate sensors and is easy to apply to individual sensors in the field.
\end{abstract}

Keywords: growing medium, rapid calibration, soil water sensors, water content

\section{INTRODUCTION}

Coir is a popular growing medium in hydroponic crop production in South Africa and worldwide (Van der Westhuizen, 2009). Its popularity can be ascribed to improved yields achieved through the use of this medium (Colla et al., 2003; Halmann and Kobryń, 2003). However, coir varies greatly from soil and other growing mediums with regard to water availability, and therefore knowledge on irrigation scheduling in coir is limited and irrigation usually managed poorly. Research by Van der Westhuizen (2009) indicated that irrigation scheduling through capacitance sensors in coir can maintain and even increase yield of hydroponic cucumber and tomato crops, while water use efficiency is greatly improved. It is however important that the water sensors are calibrated specifically for the coir.

Calibration procedures for soil water sensors have been established with the development of these sensors, but there has been very little improvement of these procedures over the years, although sensor technology has changed rapidly and continuously. Starr and Paltineanu (2002) and Cobos and Chambers (2010) detail the calibration procedures for the field and laboratory. Van der Westhuizen and Van Rensburg (2011) highlight some of the major issues encountered with these standard field and laboratory calibration procedures, of which the most critical factors are labour- and time-intensiveness due to the need for destructive sampling, as well as the waiting period required to reach the desired soil water content, and spatial variation in the field or repacking of soil in the laboratory

To whom all correspondence should be addressed.

+27 51 401-2957; e-mail: vrensbl.sci@ufs.ac.za

Received 27 August 2012; accepted in revised form 4 October 2013.
(Starr and Paltineanu, 2002; Lane and Mackenzie, 2001). The main concern for the standard field and laboratory calibration procedures are that a calibration curve is developed from very few data points. This results in calibration equations derived from single data points which, in addition, do not consider sensor response over time. All of the above-mentioned factors may create concern with regard to the precision and accuracy of calibration.

A new and improved calibration procedure for capacitance sensors in coir, that addresses these problems, has been proposed in detail by Van der Westhuizen and Van Rensburg (2011). Briefly, the method comprises the continuous gravimetric measurement of water content with load cells, as well as the continuous measurement of capacitance sensor output during a drying cycle. The drying cycle is driven by the evaporative demand of the environment as well as the water retention characteristics of the growing medium, in this case coir. Excellent precision fits for sensor response versus volumetric water content $\left(\vartheta_{v}\right)$ were achieved, and water content was predicted with high accuracy (Van der Westhuizen and Van Rensburg, 2011). The calibration procedure of Van der Westhuizen and Van Rensburg (2011) was found to be less labour-intensive, and much more accurate because of an abundance of data points. However, it still requires an extended saturation and drying period, which remains time-consuming, while it may also require some specialised equipment that is not always available to crop producers.

Soil water sensor technology is being used more frequently in commercial crop production because of good results achieved in soil (Thompson et al., 2007). However, although new and improved calibration procedures further improve the accuracy of water content management, it has become necessary to find a balance between the accuracy of calibration for 
research purposes and scientifically sound simplicity of calibration, even if slightly less accurate. Development of a simplified and rapid calibration procedure may promote the use of capacitance sensors by commercial hydroponic crop producers for irrigation management in coir. Therefore, the objectives of this study were (i) to propose a rapid procedure for calibrating $\mathrm{ECH}_{2} \mathrm{O}$ capacitance water sensors, and (ii) to evaluate the rapid calibration method for use in coir.

\section{MATERIALS AND METHODS}

Sixteen $\mathrm{ECH}_{2} \mathrm{O}$ capacitance probes (eight EC-10 and eight EC-20, Decagon devices, Inc.) were used in this experiment, of which eight were used to develop the laboratory calibration equation for each sensor type, while the remaining eight were used to evaluate the rapid procedure. The probe dimensions for EC-10 are $14.5 \mathrm{~cm} \times 3.17 \mathrm{~cm} \mathrm{x} 0.15 \mathrm{~cm}$ and for EC-20 are $25.4 \mathrm{~cm} \times 3.17 \mathrm{~cm} \times 0.15 \mathrm{~cm}$.

The sensors used to determine the laboratory calibration equations were calibrated through the continuous measurement of weight loss of a saturated coir sample during a drying cycle of at least 1 week. A detailed discussion of the laboratory procedure used for the calibration of capacitance sensors in coir can be found in Van der Westhuizen and Van Rensburg (2011). Briefly, the procedure comprises the packing of eight coir samples in eight perforated cylinders at a known bulk density $\left(100 \mathrm{~kg} \cdot \mathrm{m}^{-3}\right)$ containing one EC10 and one EC-20 sensor each. All cylinders were saturated by submerging them in deionised water. Each cylinder was suspended from a load cell, placed inside a temperature-controlled chamber directly after saturation, and drying performed through evaporation. A data logger, model CR10X of Campbell Scientific, recorded weight loss measured from the load cells and millivolt $(\mathrm{mV})$ readings from the $\mathrm{ECH}_{2} \mathrm{O}$ sensors. The $\vartheta_{v}$ within a cylinder at any given time was determined by dividing the mass of the water by the dry mass of the growing medium, and multiplying this with the bulk density of the coir (Hillel, 2004). The response (mV) of individual sensors was related to the $\vartheta_{v}$ of the growing medium, and a $4^{\text {th }}$ degree polynomial curve was fitted over the combined sensor data for both EC-10 and EC-20 sensors.

Sensor response $(x)$ and measured $\vartheta_{v}$ values $(y)$ were converted to relative sensor response $\left(x_{R e l}\right)$ and relative $\vartheta_{v}$ values $\left(y_{R e l}\right)$ by the following equations:

$$
\begin{aligned}
& \mathrm{x}_{\mathrm{Rel}}=\mathrm{x} / \mathrm{x}_{\mathrm{fc}} \\
& \mathrm{y}_{\mathrm{Rel}}=\mathrm{y} / \mathrm{y}_{\mathrm{fc}}
\end{aligned}
$$

where:

$$
\begin{aligned}
& x_{f c} \text { is the sensor response at field capacity (FC) } \\
& y_{f c} \text { is the } \vartheta_{v} \text { at FC }
\end{aligned}
$$

The relationship between relative $\vartheta_{v}$ and the average relative response of all the sensors was used to find the best fit for the data.

\section{Statistical analysis}

Volumetric water content predicted from the manufacturer's equations, and the coir-specific laboratory-determined calibration equations were compared using statistical analysis. Statistical analysis comprised the determination of the root mean squared error (RMSE), the index of agreement or D-index (Willmott, 1982) and the regression coefficient $\left(R^{2}\right)$. The following formula was used to calculate the RMSE (Willmott, 1982):

$$
R M S E=\sqrt{\frac{\sum_{i=1}^{n}\left(P_{i}-O_{i}\right)^{2}}{n}}
$$

where:

$$
\begin{aligned}
& P_{i}=\text { predicted } \\
& O_{i}=\text { observed } \\
& n=\text { sample size }
\end{aligned}
$$

The RMSE indicates the absolute fit of the model to the data, i.e., how close the observed data points are to the model's predicted values, or, in other words, how accurately the model predicts the response. Willmott (1982) used the RMSE to determine the D-index:

$$
d=1-\left[\frac{\sum_{i=1}^{n}\left(P_{i}-O_{i}\right)^{2}}{\sum_{i=1}^{n}\left(\left|P_{i}^{\prime}\right|+O_{i}^{\prime}\right)^{2}}\right]
$$

where:

$$
\begin{aligned}
P_{i} & =\text { predicted } \\
O_{i} & =\text { observed } \\
n & =\text { sample size } \\
P_{i}^{\prime} & =P_{i}-\overline{\mathrm{O}},=O_{i}-\overline{\mathrm{O}} \\
\bar{P} & =\text { mean of predicted } \\
\overline{\mathrm{O}} & =\text { mean of observed }
\end{aligned}
$$

The D-index indicates the accuracy of prediction compared to a 1:1 line, while the $R^{2}$ values only give an indication of the goodness of fit of the model (precision with which data points lie on the fitted regression line). For a good fit the $\mathrm{D}$-index and $R^{2}$ values should approach 1, while lower values of RMSE indicate better fit.

\section{RESULTS AND DISCUSSION}

\section{General principles}

Most calibration procedures are too elaborate, time-consuming and complicated for practical use in greenhouses. The proposed rapid procedure is based on the measurement of one sensor reading $(\mathrm{mV})$ and the consequent gravimetric sampling at FC, for the determination of $\vartheta_{v}$ for coir under real-time production conditions. These two measurements were used to determine a correction factor for a general calibration equation developed for coir through an accurate laboratory calibration procedure.

\section{Sensor response}

Variation in the relationship between $\vartheta_{v}$ and sensor response $(\mathrm{mV})$ exists for both EC-10 and EC20 capacitance sensors, which will result in error should a general calibration equation be derived from various individual calibration equations (Fig. 1). This variation between sensors is not unique to this experiment and is commonly experienced by other researchers (Ould Mohamed et al., 1997; Chanzy et al., 1998; Seyfried and Murdock, 2001; Bandaranayake et al., 2007; Morel et al., 2008).

The difference between sensors in this study is mainly ascribed to errors in the saturation of the coir and therefore also, to a lesser extent, to the packing of the growing medium in the calibration column. Suspecting that variation in the saturation and packing of coir will always be a problem in the commercial use of these sensors, sensor response $(\mathrm{mV})$ and measured $\vartheta_{v}$ are converted to relative sensor response $\left(x_{R e l}\right)$ and relative $\vartheta_{\mathrm{v}}\left(y_{R e l}\right)$ in an effort to reduce variation between individual sensors. This comprises of dividing both sensor response $(x)$ and measured $\vartheta_{v}(y)$ by their respective maximum values at 

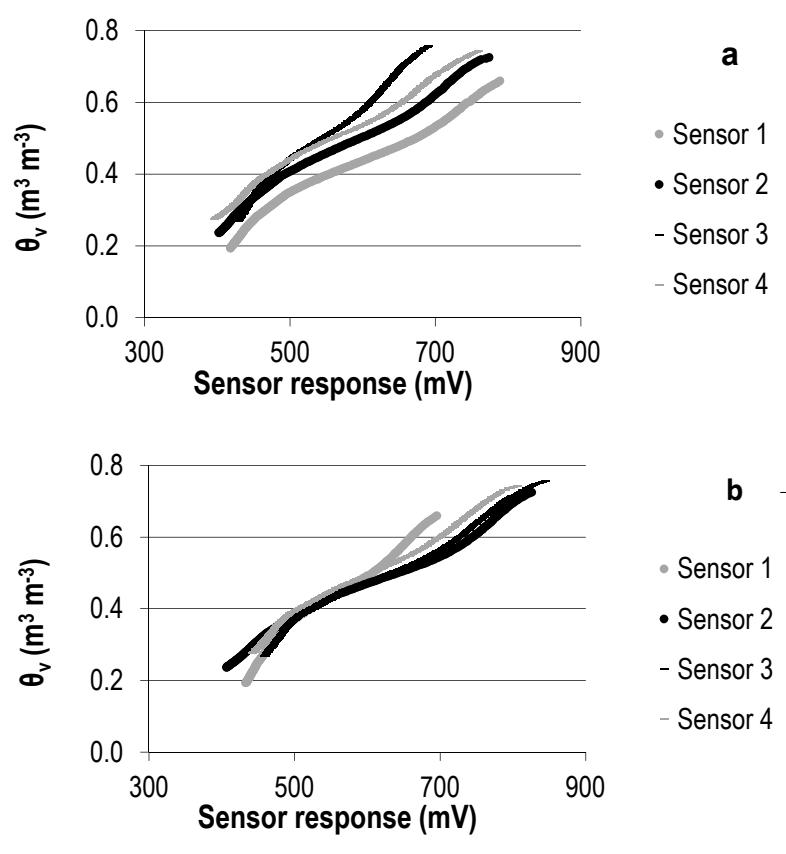

Figure 1

Graphs showing the relationship between measured volumetric water content $(\vartheta)$ and sensor response $(m V)(n=252)$ for (a) four EC-10, and (b) four EC-20 capacitance sensors.
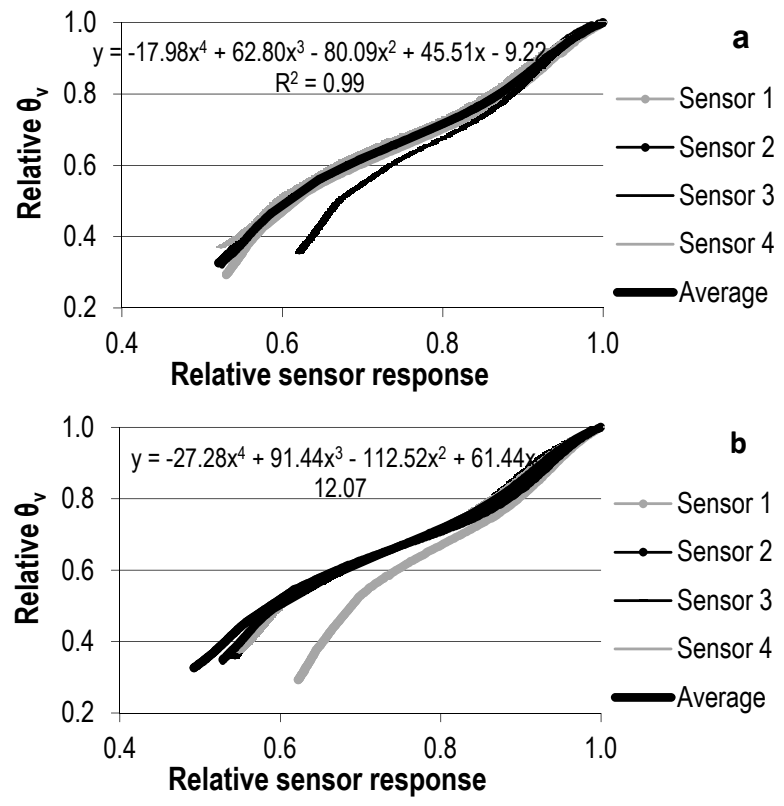

Figure 2

Graphs showing the relationship between relative measured volumetric water content $(\vartheta)$ and relative sensor response $(m V)(n=252)$ for (a) four EC-10, and (b) four EC-20 capacitance sensors; and the equations that describe the curves $\left(y=\nabla_{v}\right.$ and $\left.x=m V\right)$.

FC, namely $x_{f c}$ and $y_{f c}$. The sensor reading at FC is used instead of the reading at saturation, because saturation is difficult to reach without a vacuum chamber, and irrigation scheduling mostly occurs in the plant available water content range between the drained upper limit (DUL) or FC and the lower limit of the plant available water. The conversion to relative values of sensor response and measured $\vartheta_{v}$ reduced variation between sensors, although one outlier was observed for both EC10 and EC-20 sensors as seen in Fig. 2. This conversion eliminated large errors at the wet end, as experienced by Lane and Mackenzie (2001), while errors in the dry end fell outside of the water content range of coir. A $4^{\text {th }}$ degree polynomial curve, fitted over the combined sensor data for both EC10 and EC-20 sensors, remained the best fit for the accurate prediction of $y_{R e l}$ from $x_{\text {Rel }}$ (Fig. 2).

\section{Rapid calibration procedure}

The rapid method is based on the general calibration equation per sensor type (EC-10 or EC20), corrected by specific values of individual sensor output $\left(x_{f c}, \mathrm{mV}\right)$ and measured $\vartheta_{v}\left(y_{f c}\right)$ at FC. To get measurements at FC firstly requires the installation of the capacitance sensor in the growing medium where it will be used for irrigation management. Thereafter the growing medium is thoroughly wetted, the surface covered and allowed to drain for at least $48 \mathrm{~h}$. This point is considered as the DUL or FC. The sensor output is recorded from the point of wetting and the point of FC is identified as soon as the sensor readings remain near constant, after approximately $48 \mathrm{~h}$. Consequently, gravimetric sampling from the growing medium at $\mathrm{FC}$ is done and $\vartheta_{v}$ determined by multiplying gravimetric water content with the bulk density of the growing medium. Since the general calibration equation for coir is based on relative sensor output and relative $\vartheta_{v}, y$ will be equal to the general equation multiplied by $y_{f c}$ :

$$
\begin{aligned}
& y_{\mathrm{Rel}}=\mathrm{a}+\mathrm{b} x_{\mathrm{Rel}}+\mathrm{c} x_{\mathrm{Rel}}^{2}+\mathrm{d} x_{\mathrm{Rel}}^{3}+\mathrm{e} x_{\mathrm{Rel}}^{4} \\
& y=\left(\mathrm{a}+\mathrm{b} x_{\mathrm{Rel}}+\mathrm{c} x_{\mathrm{Rel}}^{2}+\mathrm{d} x_{\mathrm{Rel}}^{3}+\mathrm{e} x_{\mathrm{Rel}}^{4}\right) \times y_{\mathrm{fc}}
\end{aligned}
$$

Therefore, $x_{R e l}$ is determined from any given $x$ value $(\mathrm{mV})$ by dividing it by $x_{f c}(\mathrm{mV})$, which was measured by the sensors at FC, while $y_{f c}$ is the $\vartheta_{v}$ determined at FC.

\section{Evaluation of the rapid calibration procedure}

To evaluate the rapid calibration procedure, $x_{f c}$ and $y_{f c}$ were determined in coir for 4 independent sensors of EC10 and EC-20, as described in the previous section. From this, $x_{\text {Rel }}$ was determined for various readings of $x$ and the corresponding $y$ determined from the general calibration equation multiplied by $y_{f c}$. The predicted $\vartheta_{v}$ of coir using the proposed rapid calibration equations for the EC-10 and EC-20 sensors was compared to a 1:1 line with 4\% deviation boundaries in Figs. 3 and 4, respectively. The lower sections of one EC-10 curve (Sensor no. 2) and three EC-20 curves (Sensor no. 1, 3 and 4) underestimated $\vartheta_{v}$. However, according to the retention curve developed for coir in a previous experiment, the permanent wilting point (PWP), determined at $1500 \mathrm{kPa}$, is reached at $0.275 \mathrm{~m}^{3} \cdot \mathrm{m}^{3}$, meaning that this underestimation will not influence the prediction of $\vartheta_{v}$ in the available water content range for coir. Deviation from the 1:1 line at the dry end, predicted by TDR, as experienced by Chanzy et al. (1998), also fell outside of the available water content range for the soil used and therefore was not significant for the purpose of irrigation management within the available water content range.

The figures clearly indicate that the proposed rapid calibration for coir is reasonably accurate, especially between DUL and PWP, for most EC-10 and EC-20 sensors. To verify the relative reliability of the rapid procedure, statistical analysis was done separately for all data points and for data points between DUL and PWP. 

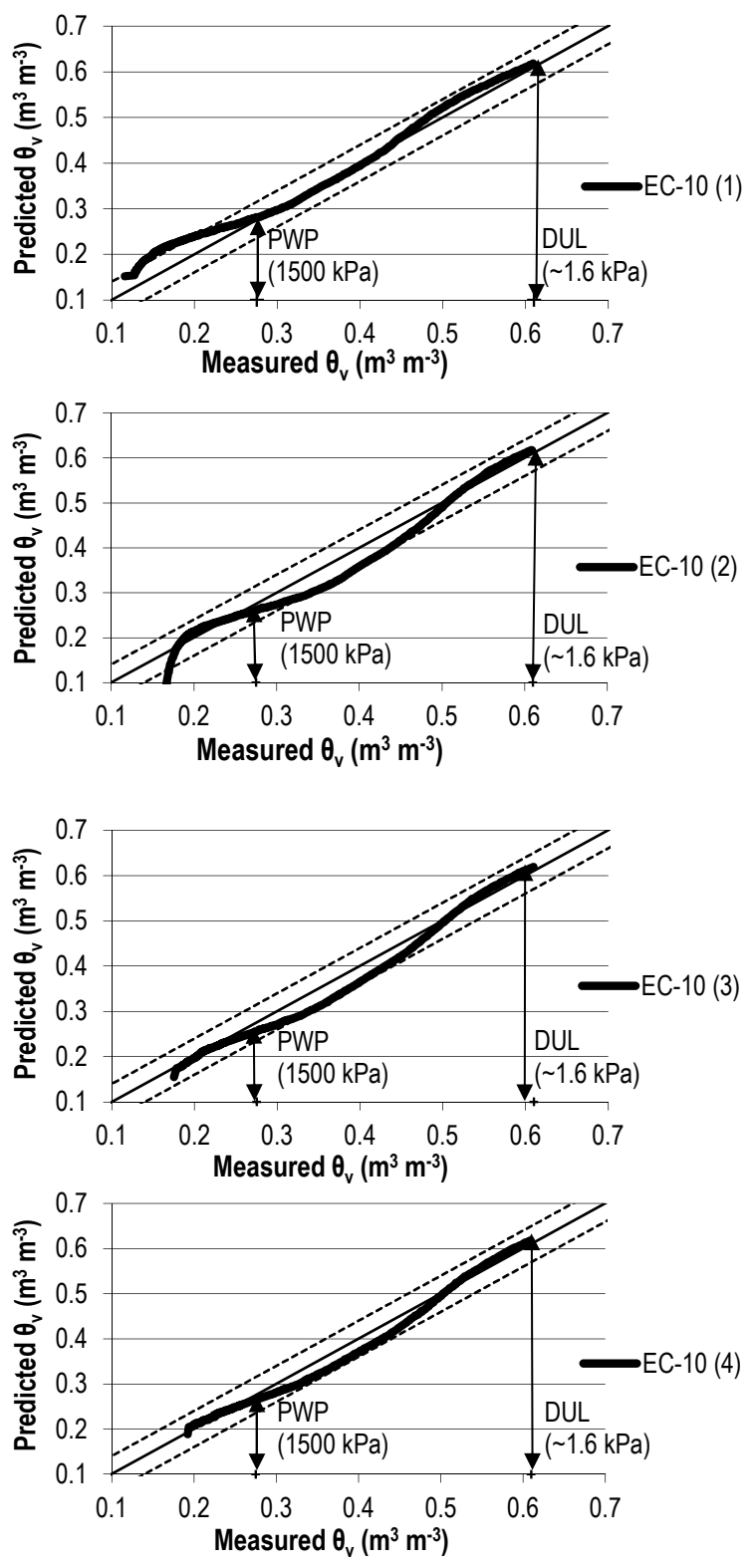

Figure 3

Graphs showing the relationship between measured volumetric water content $\left(\vartheta_{v}\right)(n=252)$ and $\nabla_{v}$ predicted using the rapid calibration procedure for four EC-10 capacitance sensors, EC-10 (1) to (4). The 1:1 line, specified $4 \%$ accuracy boundary lines on opposite sides of this line, drained upper limit (DUL) and permanent wilting point (PWP) for coir are also presented.

The accuracy of the proposed rapid calibration procedure proved to be equally reliable over all data points and for data points between DUL and PWP for all EC-10 sensors: the Dindex approached 1; $R^{2}$ was 0.97 or better (Table 1). RMSE varied between 0.012 and $0.030 \mathrm{~m}^{3} \cdot \mathrm{m}^{-3}$ for the prediction of $\vartheta_{v}$ between DUL $\left(0.607 \mathrm{~m}^{3} \cdot \mathrm{m}^{-3}\right)$ and PWP $\left(0.275 \mathrm{~m}^{3} \cdot \mathrm{m}^{-3}\right)$ with the proposed rapid calibration procedure.

Compared to all data points, the accuracy of the proposed rapid calibration procedure for data points between DUL and PWP proved to be more reliable for all EC-20 sensors: the Dindex approached $1 ; R^{2}$ was 0.99 or better (Table 1). RMSE varied between 0.014 and $0.021 \mathrm{~m}^{3} \cdot \mathrm{m}^{3}$ for the prediction of $\vartheta_{v}$ between DUL $\left(0.607 \mathrm{~m}^{3} \cdot \mathrm{m}^{-3}\right)$ and PWP $\left(0.275 \mathrm{~m}^{3} \cdot \mathrm{m}^{-3}\right)$ with the proposed rapid calibration procedure.
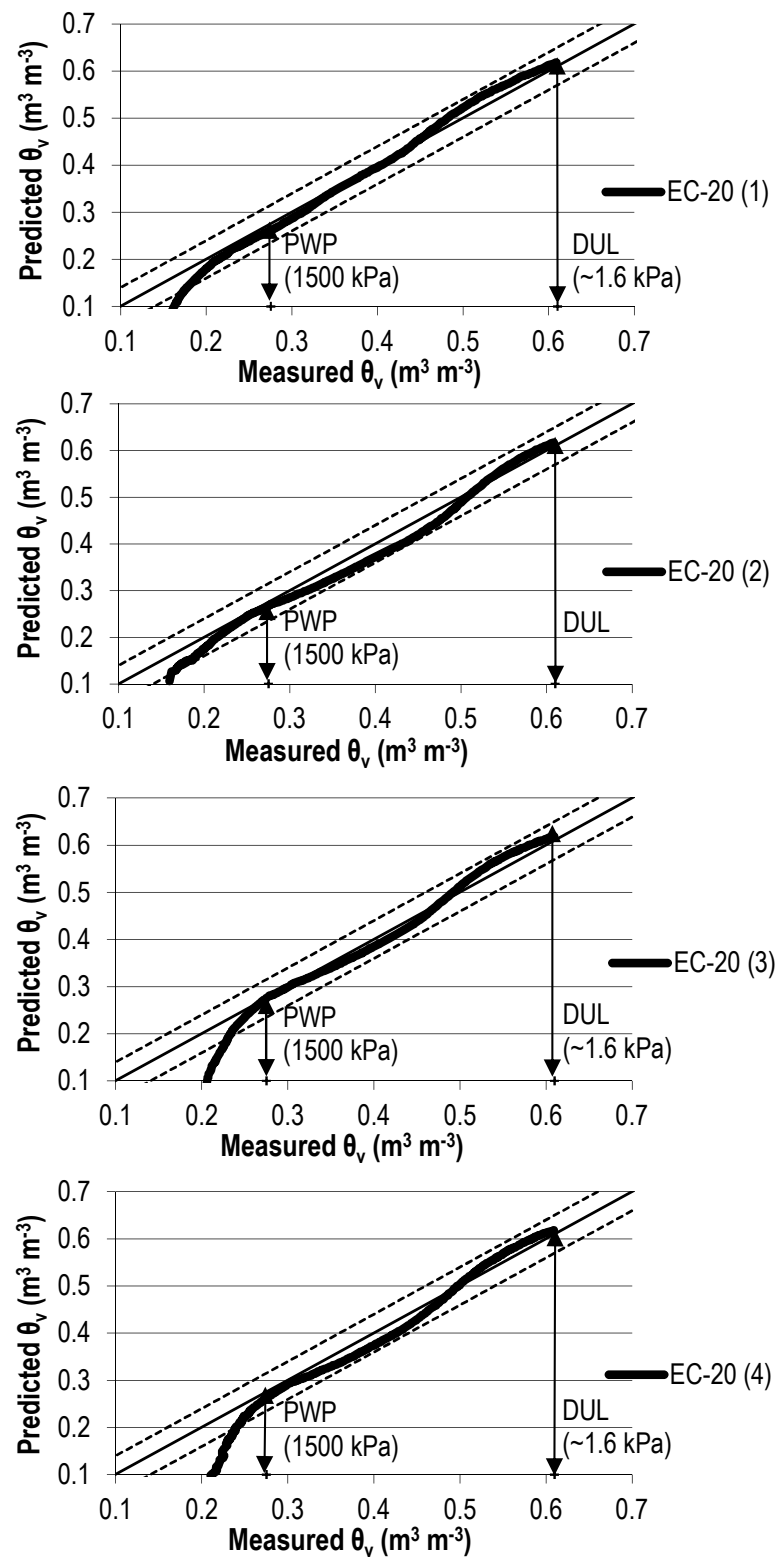

Figure 4

Graphs showing the relationship between measured volumetric water content $\left(\vartheta_{v}\right)(n=252)$ and $\mathbb{\bigotimes}_{v}$ predicted using the rapid calibration procedure for four EC-20 capacitance sensors, EC-20 (1) to (4). The 1:1 line, the specified $4 \%$ accuracy boundary lines on opposite sides of this line, drained upper limit (DUL) and permanent wilting point (PWP) for coir are also presented.

In contrast, the comparable values for all data points for the EC-20 sensors were as follows: RMSE varied between 0.021 and 0.066 ; the $\mathrm{D}$-index varied between 0.95 and $0.99 ; R^{2}$ varied between 0.94 and 0.99 (Table 1).

The statistical results in Table 1 show that the proposed rapid calibration procedure yielded a good prediction of the $\vartheta_{v}$ by EC-20 for data points between DUL and PWP, while the statistical results were similar for the prediction of $\vartheta_{v}$ for all data points and data points between DUL and PWP by EC-10. Since the $4 \%$ accuracy of prediction for EC-10 and EC-20 sensors indicated by the manufacturer is based on more accurate and time-consuming calibration procedures, slight deviation from these boundaries may be acceptable when compared to the benefit of using the rapid calibration 
TABLE 1

Statistical analysis of all data points and data points between the drained upper limit (DUL) and permanent wilting point (PWP) for all sensors of EC-10 and EC-20

\begin{tabular}{|l|c|c|c|c|c|c|}
\hline Sensor & \multicolumn{3}{|c|}{ All data points } & \multicolumn{3}{c|}{$\begin{array}{c}\text { Data points between } \\
\text { DUL and PWP }\end{array}$} \\
\cline { 2 - 7 } & D-Index & $\boldsymbol{R}^{\mathbf{2}}$ & RMSE & D-Index & $\boldsymbol{R}^{\mathbf{2}}$ & RMSE \\
\hline EC-10: & & & & & & \\
Sensor 1 & 0.99 & 0.99 & 0.030 & 1.00 & 1.00 & 0.012 \\
Sensor 2 & 0.99 & 0.97 & 0.026 & 0.98 & 0.99 & 0.030 \\
Sensor 3 & 0.99 & 0.98 & 0.022 & 0.98 & 0.99 & 0.027 \\
Sensor 4 & 1.00 & 0.99 & 0.018 & 0.99 & 0.99 & 0.021 \\
& & & & & & \\
EC-20: & & & & & & \\
Sensor 1 & 0.97 & 0.97 & 0.059 & 1.00 & 1.00 & 0.014 \\
Sensor 2 & 0.99 & 0.99 & 0.021 & 0.99 & 0.99 & 0.021 \\
Sensor 3 & 0.95 & 0.94 & 0.066 & 1.00 & 0.99 & 0.014 \\
Sensor 4 & 0.97 & 0.97 & 0.051 & 0.99 & 0.99 & 0.018 \\
\hline
\end{tabular}

procedure in the field and the ease of applying it to individual sensors.

\section{CONCLUSIONS}

A simple procedure was developed and tested for the rapid calibration of $\mathrm{ECH}_{2} \mathrm{O}$ (EC-10 and EC-20) capacitance sensors in their operational environment. The method is based on the taking of only one sensor reading and one gravimetric sample, both at FC and in the environment in which they will be used to manage irrigation, to determine a correction factor for an accurate general laboratory calibration equation developed for coir.

The general calibration equation for coir was obtained for half of the sensors through an accurate laboratory calibration procedure. Great variation between sensors, with regard to the relationship between sensor output and $\vartheta_{v}$, posed the problem of high percentages of over- and/or underestimation of water content if data for the individual sensors were to be combined into a calibration equation for each sensor type (EC-10 and EC-20). Sensor response and measured $\vartheta_{v}$ were converted to relative sensor response and relative $\vartheta_{v}$ by dividing both sensor response and measured $\vartheta_{v}$ with their respective maximum values at FC. This conversion reduced sensor-to-sensor variation. A perfect fit, indicated by $R^{2}$ values greater than 0.99 for the relationship between relative sensor response and relative $\vartheta_{v}$ was achieved by a $4^{\text {th }}$ degree polynomial curve for both EC-10 and EC-20 sensors in coir. The equation consequently incorporated the measured sensor response and measured $\vartheta_{v}$ at FC in operational conditions in the greenhouse.

The calibration equations for EC-10 and EC-20 were evaluated with different sensors, of which only the sensor output and $\vartheta_{v}$, determined through gravimetric sampling were measured at FC for each sensor. Various methods used for statistical evaluation of the rapid calibration pointed to a good accuracy for the prediction of $\vartheta_{v}$ between DUL and PWP, for both EC-10 and
EC-20 sensors in coir. The rapid method, based on a growing medium-specific calibration with relative values of sensor output and measured $\vartheta_{v}$, should perform equally well in other growing mediums. In conclusion, it must be emphasised that individual sensor calibration is essential for the accurate prediction of $\vartheta_{v}$, while the rapid calibration procedure for $\mathrm{ECH}_{2} \mathrm{O}$ capacitance sensors will simplify their use in commercial greenhouses, for the purpose of improved irrigation management in coir.

\section{REFERENCES}

BANDARANAYAKE WM, PARSONS LR, BORHAN MS and HOLETON JD (2007) Performance of a capacitance-type soil water probe in a well-drained sandy soil. Soil Sci. Soc. Am. J. 71 993-1002.

CHANZY A, CHADOEUF J, GAUDU JC, MOHRATH D, RICHARD G and BRUCKLER L (1998) Soil moisture monitoring at the field scale using automatic capacitance probes. Eur. J. Soil Sci. 49 637-648.

COBOS DR and CHAMBERS C (2010) Calibrating ECH $\mathrm{E}_{2} \mathrm{O}_{\text {soil mois- }}$ ture sensors. Application note. URL: http://www.decagon.com/ assets/Uploads/13393-04-CalibratingECH2OSoilMoistureProbes. pdf (Accessed 24 March 2012).

COLLA G, SACCARDO F, REA E, PIERANDREI F and SALERNO A (2003) Effects of substrates on yield, quality and mineral composition of soilless-grown cucumbers. Acta Hort. 614 205-209.

HALMANN E and KOBRYŃ J (2003) Yield and quality of cherry tomato (Lycopersicon esculentum var. cerasiforme) cultivated on rockwool and cocofibre. Acta Hort. 614 693-697.

HILLEL D (2004) Introduction to Environmental Soil Physics. Elsevier Science, Massachusetts.

LANE PNJ and MACKENZIE DH (2001) Field and laboratory calibration and test of TDR and capacitance techniques for indirect measurement of soil water content. Aust. J. Soil Res. 39 1371-1386.

MOREL P, GUILLEMAIN G and MICHEL J-C (2008) Temperature and salinity effects on measurements of growing media moisture content carried out with TDR and capacitance probes. Acta Hort. 779 393-399.

OULD MOHAMED S, BERTUZZI P, BRUAND A, RAISON L and BRUCKLER L (1997) Field evaluation and error analysis of soil water content measurement using the capacitance probe method. Soil Sci. Soc. Am. J. 61 399-408.

SEYFRIED MS and MURDOCK MD (2001) Response of a new soil water sensor to variable soil, water content and temperature. Soil Sci. Soc. Am. J. 65 28-34.

STARR JL and PALTINEANU IC (2002) The soil solution phase. In: Dane JH and Topp GC (eds.) Methods of Soil Analysis, Part 4, Physical Methods. SSSA, Madison. 463-474.

THOMPSON RB, GALLARDO M, VALDEZ LC and FERNÁNDEZ MD (2007) Determination of lower limits for irrigation management using in situ assessments of apparent crop water uptake made with volumetric soil water content sensors. Agric. Water Manage. 92 13-28.

VAN DER WESTHUIZEN RJ (2009) Irrigation scheduling of tomatoes (Lycopersicon esculentum Mill.) and cucumbers (Cucumis sativus L.) grown hydroponically in coir. $\mathrm{PhD}$ thesis, Department of Agronomy, Stellenbosch University.

VAN DER WESTHUIZEN RJ and VAN RENSBURG LD (2011) A laboratory procedure for the calibration of EC-10 and EC-20 capacitance sensors in coir. Europ. J. Hort. Sci. 76 (4) 151-157.

WILLMOTT CJ (1982) Some comments on the evaluation of model performance. Bull. Am. Meteorol. Soc. 63 1309-1313. 
http://dx.doi.org/10.4314/wsa.v39i5.18 Available on website http://www.wrc.org.za

ISSN 0378-4738 (Print) = Water SA Vol. 39 No. 5 October 2013 ISSN 1816-7950 (On-line) = Water SA Vol. 39 No. 5 October 2013 